\title{
Efficient Radio Map Construction Based on Low-Rank Approximation for Indoor Positioning
}

\author{
Yongli Hu, Wei Zhou, Zheng Wen, Yanfeng Sun, and Baocai Yin \\ Beijing Key Laboratory of Multimedia and Intelligent Software Technology, College of Computer Science and Technology, \\ Beijing University of Technology, Beijing 100124, China
}

Correspondence should be addressed to Yanfeng Sun; yfsun@bjut.edu.cn

Received 4 July 2013; Accepted 5 September 2013

Academic Editor: Matjaz Perc

Copyright (C) 2013 Yongli Hu et al. This is an open access article distributed under the Creative Commons Attribution License, which permits unrestricted use, distribution, and reproduction in any medium, provided the original work is properly cited.

Fingerprint-based positioning in a wireless local area network (WLAN) environment has received much attention recently. One key issue for the positioning method is the radio map construction, which generally requires significant effort to collect enough measurements of received signal strength (RSS). Based on the observation that RSSs have high spatial correlation, we propose an efficient radio map construction method based on low-rank approximation. Different from the conventional interpolation methods, the proposed method represents the distribution of RSSs as a low-rank matrix and constructs the dense radio map from relative sparse measurements by a revised low-rank matrix completion method. To evaluate the proposed method, both simulation tests and field experiments have been conducted. The experimental results indicate that the proposed method can reduce the RSS measurements evidently. Moreover, using the constructed radio maps for positioning, the positioning accuracy is also improved.

\section{Introduction}

Recently, with the rapidly increasing location-based services (LBS), such as positioning, tracking, navigation, and location-based security, the positioning issue has been extensively studied. In an indoor environment, the wireless local area network (WLAN) is considered to be a basic infrastructure and can be easily implemented because of its ubiquitous coverage. For a WiFi-integrated mobile device, its location can be determined using the received signal strength (RSS) by many positioning methods $[1,2]$, in which the fingerprintbased method is regarded as a potential method with acceptable positioning accuracy.

Generally, the fingerprint-based positioning method is implemented in two phases: the off-line training phase and the online positioning phase. In the off-line training phase, at the selected reference points, the RSSs from different access points (APs) are measured by a mobile device. The RSS measurements and the correspondent locations are generally formulated as the radio map, which infers the relation between the RSS distribution and the spatial locations. In the online positioning phase, an observed RSS measurement is matched to the radio map, and the location can be estimated by many proposed methods, such as $k$-nearest neighbor algorithm (KNN) [1], kernel-based algorithm [3], or Bayesian estimation method [4].

The radio map is vital for the fingerprint-based positioning method, but the construction of a radio map is a timeconsuming work. To get complete RSS samples in the target area, the measurements should be recorded at dense reference points. However, in a complicated indoor environment, it is impractical to get RSSs anywhere because of the limitations of building structure and no admittance to some rooms. So some researchers attempt to reduce the measurements of radio map construction and estimate the unknown measurements using radio propagation model or interpolation methods. Compared with the intensive researches on the positioning methods, less attention has been paid to the fundamental work of radio map construction. How to construct the radio map efficiently is still a challenging issue for the fingerprint-based positioning.

In this paper, we propose an efficient radio map construction method based on low-rank approximation. Different from the existing interpolation methods, the proposed method represents the RSS distribution as a low-rank matrix based on two observations. For one thing the RSS data has 
high spatial correlation which implies low-rank property; for another, as an extension of compressive sensing theory, the low-rank approximation is a proven method in data reconstruction with high efficiency and good performance $[5,6]$. So based on the low-rank matrix complement model [7], we construct the RSSs radio map from relatively sparse measurements. Additionally, to eliminate the interference of noises and obtain practical results, the basic low-rank complement model is revised by combining it with the signal spatial consistency, namely, a smoothing low-rank (SLR) model. The proposed method is evaluated on both simulation and field test data compared with the previous methods. The results show that the measurements of radio map construction can be significantly reduced and the positioning accuracy is also improved using the constructed radio map for positioning. The novelty of this paper is that we represent the RSS radio map construction problem as a revised low-rank matrix complement model. Additionally, the proposed optimization solution to the SLR model is another contribution of this paper.

The remainder of this paper is organized as follows. In Section 2, the related work of RSS radio map construction methods is briefly described. In Section 3, the low-rank complement theory and the details of the proposed SLR model are presented. In Section 4, the proposed radio map construction method is evaluated and the positioning experiments with the constructed radio map are implemented. The conclusions are drawn in Section 5.

\section{Related Work}

Constructing a fingerprint radio map is an essential step of positioning and has great influence on the positioning accuracy. Generally, high positioning accuracy can be obtained by a dense radio map. To achieve dense fingerprint measurements and reduce the calibration efforts, inserting some virtual data from the real measurements is a direct way. Many researchers utilize the radio propagation model to get dense measurements. For example, Xiang et al. [8] and Kuo and Tseng [9] use the radio propagation model to construct the radio map from a small number of on-site signal measurements. Due to the diversity of indoor environments, the common radio propagation model cannot model the RSS multipath fading and interference. So the interpolation methods, such as radial basis function (RBF) [10] and inverse distance weighting (IDW) [9], are used to construct a dense radio map. These methods generally estimate the missing RSS data by the interpolation of the measurements at local adjacent reference points. The measurements at local neighborhood usually have correlative relation. Therefore, the positioning accuracy of the extended radio map by interpolation usually outperforms the result of the original radio map.

Although the performance of the interpolation methods is acceptable, it still demands too much labeled data for obtaining accurate positioning results. So some new explorations are made to realize positioning with less RSS measurements. For example, considering the signal sparsity, the compressive sensing (CS) theory is used for indoor positioning systems [11]. Inspired by this work, we utilize the low-rank approximation method to reduce the effort of constructing the fingerprint radio map. As an extension of the CS theory, low-rank matrix representation is considered more suitable for modeling the RSS spatial distribution because the signal sparse property is described in the inherent 2D space, while CS only describes the signal sparsity in the transformation coefficient domain. However, the low-rank matrix completion problem and its solution are intensively discussed recently [7, 12-14]. Through proper sampling, the low-rank optimization can exactly recover the whole matrix merely from a small number of entries. So the low-rank completion method is widely used in many applications. For example, in WSNs, Zhang et al. [15] present a novel approach using the low-rank approximation to estimate the missing values in traffic matrices based on nuclear norm minimization. Cheng et al. [6] propose an efficient data collection approach based on a low-rank completion technique. Nikitaki et al. [16] present an efficient fingerprint positioning training method using matrix completion, which is the most related work to our method and can be regarded as the basic low-rank (BLR) model in our paper. However, in their method, the spatial consistence of RSSs is not considered and the solution of the matrix complement model is not discussed. Additionally, the validation of their method is only verified by simulation data. So we will continue to explore the application of low-rank completion method in RSS radio map construction and propose a revised low-rank complement model.

\section{Methods}

3.1. Basic Low-Rank Matrix Completion Model. If the positioning area is represented as a rectangle, the regular RSS measurements at $N_{1} \times N_{2}$ reference points will generate an $N_{1} \times N_{2}$ matrix for each AP. If the sampling rate of RSS measurement is dense enough, the positioning will get high accuracy. However, it is impractical to get complete measurements at every reference point in the dense grid. Usually the RSS value at a small subset of the reference point can be collected, so the RSS measurement matrix is often incomplete. Here we adopt a mask operator $\mathscr{A}(\cdot)$ to represent the subset sampling procedure:

$$
\mathscr{A}(X)=B \text {, }
$$

where $X$ is the complete measurement matrix at all reference points and the element $X(i, j)$ represents the RSS value of an $\mathrm{AP}$ at the reference point $(i, j) . B$ is an incomplete matrix with only a sparse set of elements having valid values, which means the RSS values at these reference points are available. For the sake of clarity, the operator $\mathscr{A}(\cdot)$ can be specified as an $N_{1} \times N_{2}$ matrix $Q$, such that

$$
Q(i, j)= \begin{cases}1 & \begin{array}{l}
\text { if the reference point }(i, j) \\
\text { has valid RSS value }
\end{array} \\
0 & \text { otherwise }\end{cases}
$$


By this formulation, $\mathscr{A}(X)=Q * X=B$, where $*$ represents an element-wise product; that is, $B(i, j)=Q(i, j) X(i, j)$.

As the RSS signal distribution has highly spatial and temporary correlation, the measurement matrix $X$ collected at the target area is considered as having low-rank property approximately. Recently, Candes and Recht have proved that it is highly possible to recover a low-rank matrix from a subset of its entries [7]. Thus, we can recover the measurement matrix by solving the following optimization problem:

$$
\begin{aligned}
& \min \operatorname{rank}(X), \\
& \text { subject to } \mathscr{A}(X)=B .
\end{aligned}
$$

This is the basic low-rank matrix completion model for fingerprint radio map construction.

\subsection{Smoothing Low-Rank Matrix Approximation Model.} Although the low-rank matrix completion method is widely used for data reconstruction, the data properties, such as the distribution, consistence, and smoothness, are not well specified in practice. Using the basic low-rank model, the reconstruction error is usually little at the known reference points, but the reconstruction matrix is not consistent. However, the actual RSS signal spatial propagation is normally consistent at local area except for some noisy or singular measurements. To obtain a practical and local continuous reconstructed matrix, we propose a smoothing low-rank matrix approximation model by adding the smoothness constraint term in the basic low-rank model. So the low-rank model in (3) is revised as the following form:

$$
\begin{aligned}
& \min \operatorname{rank}(X)+\lambda S(X), \\
& \text { subject to } \mathscr{A}(X)=B,
\end{aligned}
$$

where $S(X)$ is the smooth term; that is, small value of $S(X)$ infers a good consistence of $X . \lambda$ is the weight to balance the low-rank term and the smooth term, which is usually determined by experiments. We define $S(X)$ via the diversity of matrix horizontal and vertical difference; that is,

$$
S(X)=\left\|\mathscr{D}_{x}(X)\right\|_{F}^{2}+\left\|\mathscr{D}_{y}(X)\right\|_{F}^{2}
$$

where $\mathscr{D}_{x}(X)$ is an $N_{1} \times\left(N_{2}-1\right)$ matrix representing the horizontal difference of $X$ with element in form of $D_{x}(i, j)=$ $X(i, j+1)-X(i, j)$, and $\mathscr{D}_{y}(X)$ is a $\left(N_{1}-1\right) \times N_{2}$ matrix representing the vertical difference of $X$ with element in form of $D_{y}(i, j)=X(i+1, j)-X(i, j)$. The operator $\|\cdot\|_{F}^{2}$ is the Frobenius norm of a matrix. From this representation, the SLR model is formulated as the following optimization:

$$
\begin{aligned}
& \min \operatorname{rank}(X)+\lambda\left(\left\|\mathscr{D}_{x}(X)\right\|_{F}^{2}+\left\|\mathscr{D}_{y}(X)\right\|_{F}^{2}\right), \\
& \text { subject to } \mathscr{A}(X)=B .
\end{aligned}
$$

3.3. Model Solution Based on Singular Value Decomposition. However, the minimization of matrix rank is not practical because it is an NP-hard problem [7]. To work out this optimization problem, the nuclear norm $\|\cdot\|_{*}$ is proposed to replace the matrix rank approximately [13]. Yet the time complexity is still high for a large dimension matrix. In this paper, a solution based on singular value decomposition (SVD) is used to solve the low-rank matrix issue. Firstly, the measurement matrix $X$ is decomposed by SVD technique into three matrices:

$$
X=U \Sigma V^{T}
$$

where $U$ is an $N_{1} \times N_{1}$ unitary matrix and $V$ is an $N_{2} \times N_{2}$ unitary matrix. $\sum$ is an $N_{1} \times N_{2}$ diagonal matrix containing the singular values $\sigma_{k}$, which are arranged in a decreasing order. Then we can factorize the matrix $X$ as $X=U \Sigma V^{T}=L R^{T}$, where $L=U \Sigma^{1 / 2}$ and $R=V \Sigma^{1 / 2}$. So the model in (6) is replaced by the following minimization model:

$$
\begin{aligned}
\min \operatorname{rank}\left(L R^{T}\right)+\lambda( & \left\|\mathscr{D}_{x}\left(L R^{T}\right)\right\|_{F}^{2} \\
& \left.+\left\|\mathscr{D}_{y}\left(L R^{T}\right)\right\|_{F}^{2}\right),
\end{aligned}
$$

subject to $\mathscr{A}\left(L R^{T}\right)=B$.

By the low-rank property of $X$, the dimensions of $L$ and $R$ can be reduced to $N_{1} \times K$ and $N_{2} \times K$, respectively, by omitting minimal singular values, where $K$ is a preestimated value which is determined by the rank of $X$. From the lemma in [17], if the restricted isometry property holds on $\mathscr{A}(X)$ and $\operatorname{rank}(X)<\operatorname{rank}\left(L R^{T}\right)$, then (8) is equivalent to the following model:

$$
\begin{aligned}
\min & \|L\|_{F}^{2}+\|R\|_{F}^{2} \\
& +\lambda\left(\left\|\mathscr{D}_{x}\left(L R^{T}\right)\right\|_{F}^{2}+\left\|\mathscr{D}_{y}\left(L R^{T}\right)\right\|_{F}^{2}\right),
\end{aligned}
$$

subject to $\mathscr{A}\left(L R^{T}\right)=B$.

Considering that the measurements received by the mobile devices are often not accurate and the matrix in our scenario does not completely have the low-rank property, we relax the condition of $\mathscr{A}\left(L R^{T}\right)=B$ and transform the constrained minimization model to an unconstrained model as the following form:

$$
\begin{aligned}
\min \|L\|_{F}^{2} & +\|R\|_{F}^{2}+\eta\left\|\mathscr{A}\left(L R^{T}\right)-B\right\|_{F}^{2} \\
& +\lambda\left(\left\|\mathscr{D}_{x}\left(L R^{T}\right)\right\|_{F}^{2}+\left\|\mathscr{D}_{y}\left(L R^{T}\right)\right\|_{F}^{2}\right),
\end{aligned}
$$

where $\left\|\mathscr{A}\left(L R^{T}\right)-B\right\|_{F}^{2}$ represents the reconstruction error at the sampling subset $B$ with an adjusting weight $\eta$. This is the final smoothing low-rank matrix completion model for fingerprint radio map construction.

To solve the minimum problem in (10), we adopt an alternant iteration algorithm for $L$ and $R$. Firstly, $L$ and $R$ are initialized randomly. Then, we fix $L$ and optimize $R$ by a linear least square method. After $R$ is updated by the optimization, we fix $R$ and let $L$ be the optimization variable. Repeat the above alternant iteration procedure until the target 


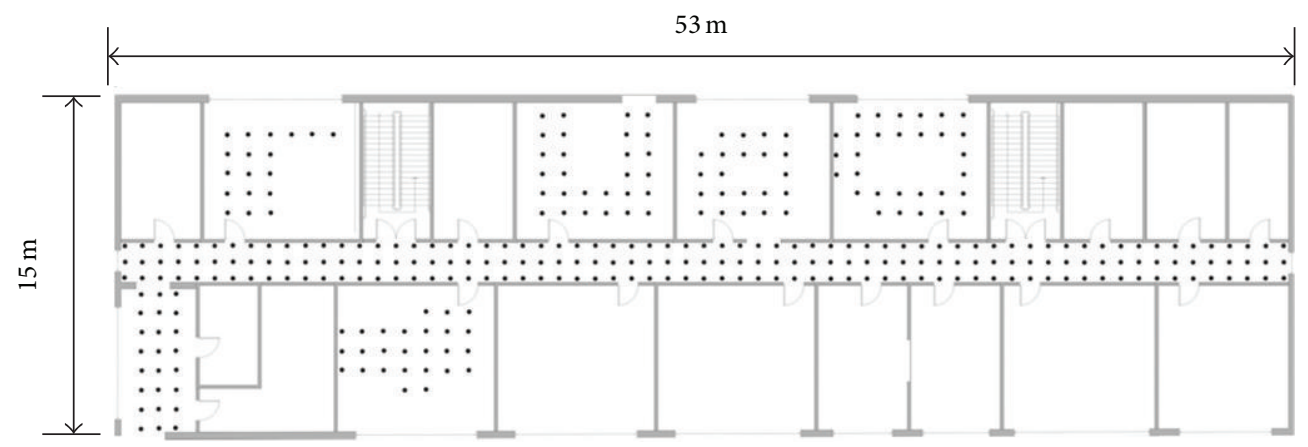

FiguRE 1: The experimental area in the third floor of the information building in Beijing University of Technology. The black points are reference points.

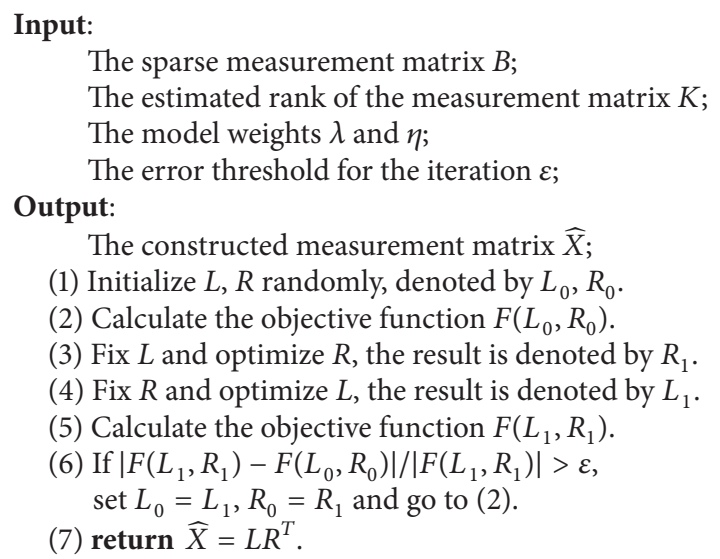

(1) Initialize $L, R$ randomly, denoted by $L_{0}, R_{0}$.

(2) Calculate the objective function $F\left(L_{0}, R_{0}\right)$.

(3) Fix $L$ and optimize $R$, the result is denoted by $R_{1}$.

(4) Fix $R$ and optimize $L$, the result is denoted by $L_{1}$.

(5) Calculate the objective function $F\left(L_{1}, R_{1}\right)$.

(6) If $\left|F\left(L_{1}, R_{1}\right)-F\left(L_{0}, R_{0}\right)\right| /\left|F\left(L_{1}, R_{1}\right)\right|>\varepsilon$, set $L_{0}=L_{1}, R_{0}=R_{1}$ and go to (2).

(7) return $\widehat{X}=L R^{T}$.

Algorithm 1: The algorithm of smoothing low-rank completion model.

function in (10), denoted by $F(L, R)$, converges and meets the preassigned error threshold $\varepsilon$. The details of the alternant iteration algorithm are shown in Algorithm 1.

\section{Results and Discussion}

4.1. Experiments Setup. To evaluate the proposed method, we implement the fingerprint radio map construction experiment both on simulated and real data, and the constructed results are compared with IDW, RBF, and the basic lowrank (BLR) method. The positioning performance of the constructed radio map is also validated in this section.

In the simulation experiment, we assume that the RSS values at 5000 reference points are collected in a $100 \mathrm{~m} \times$ $50 \mathrm{~m}$ square field with stepsize of $1 \mathrm{~m}$ in horizontal and vertical directions. We randomly deploy 50 APs in this area. To simulate the RSS signal spatial distribution, we use the following radio propagation path loss model to simulate the signal attenuation:

$$
P_{r}(d)=P_{t}-\bar{P}\left(d_{0}\right)-10 n \log _{10}\left(\frac{d}{d_{0}}\right)-X_{\sigma}
$$

where $P_{r}(d)$ is the receiving power at distance $d$ from the AP, $P_{t}$ is the transmitting power, and $\bar{P}\left(d_{0}\right)$ is the average path loss value at the reference distance $d_{0}$ (generally set to $1 \mathrm{~m}) . n$ is the path loss exponent, and $X_{\sigma}$ represents Gaussian noise with the distribution $N(0, \sigma)$. Therefore, the RSSs can be calculated if the path loss exponent is known. We assume that transmission distance is 30 meters; in other words, if the distance between the AP and the reference point is more than 30 meters, the RSS value will be set to $-100 \mathrm{~dB}$. We set the path loss exponent $n=4.4$ and the average of the path loss value $\bar{P}\left(d_{0}\right)=-35 \mathrm{~dB}$. The variance of the noise is confined into the interval $[0,16]$. Under this experiment setup, all of the RSS values between reference points and APs can be obtained. Totally, the radio map has $50 \times 100 \times 50$ measurements and each AP has $100 \times 50$ measurements, which forms an instance of the measurement matrix $X$. In the radio map construction experiments, a subset of the measurements at the $100 \times 50$ reference points (e.g., 20\%) is randomly selected for low-rank completion.

The field experiment is performed in a laboratory area with the dimension of $53 \mathrm{~m} \times 15 \mathrm{~m}$ (shown in Figure 1) in the third floor of the information building in Beijing University of Technology. In this area, about 20 APs can be detected. In the off-line RSSs collection, a person carrying a mobile device moves through the area, and the RSS value and its location are recorded. Totally at 337 reference points (the black points in Figure 1), the RSS measurements are collected. In order to avoid systematic errors and obtain a reliable radio map, at each reference point we collect 10 samples for each AP. The average of the 10 measurements is recorded as the final RSS value for this reference point. To recover the whole measurements in the test area by the proposed SLR model, we randomly select a part of the 337 reference points as the known measurements, that is, $B$ in (10). The remainder measurements at the 337 reference points are used for evaluating the constructed results.

4.2. Radio Map Construction Results. To intuitively display the radio map construction results, the radio map is rendered as an image with the color values representing the RSS measurements. In the simulation experiment, the original simulated measurements of an AP and the constructed results 


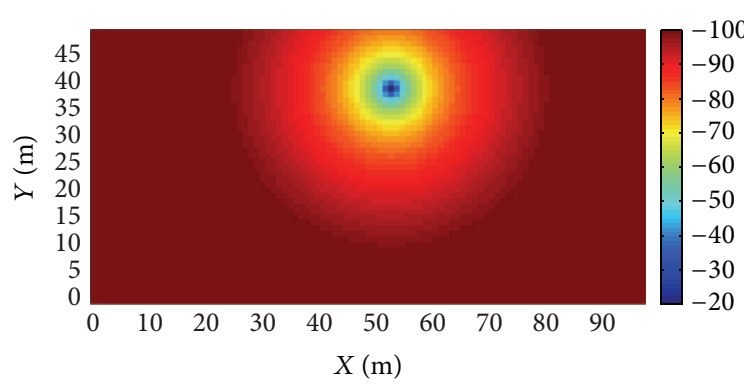

(a) Original

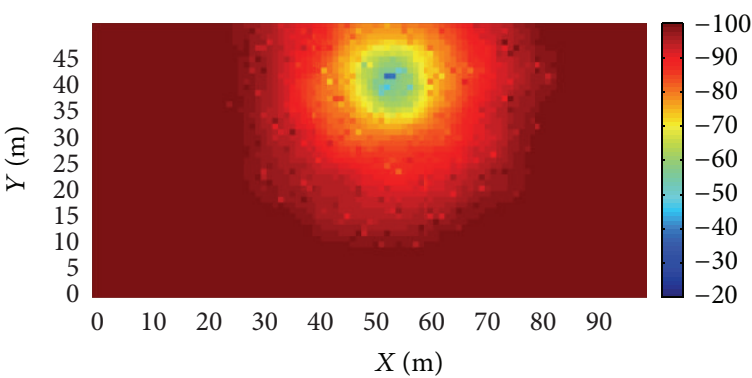

(c) IDW

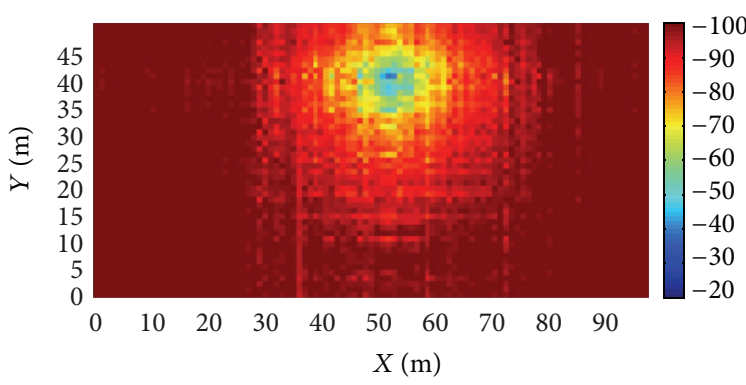

(e) BLR

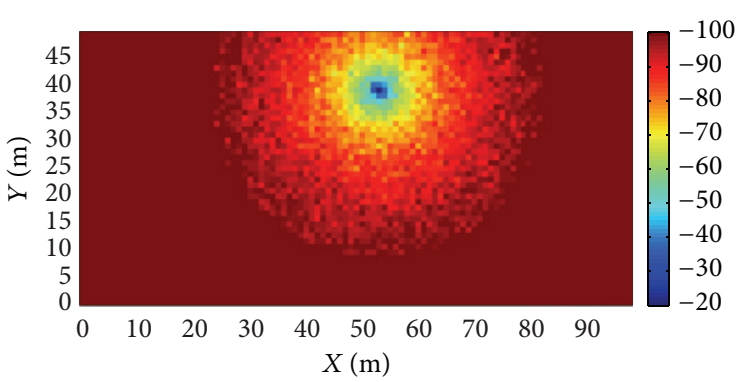

(b) Original with noise

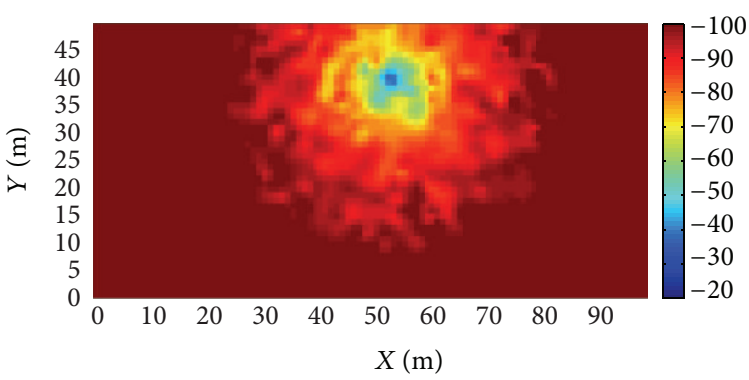

(d) $\mathrm{RBF}$

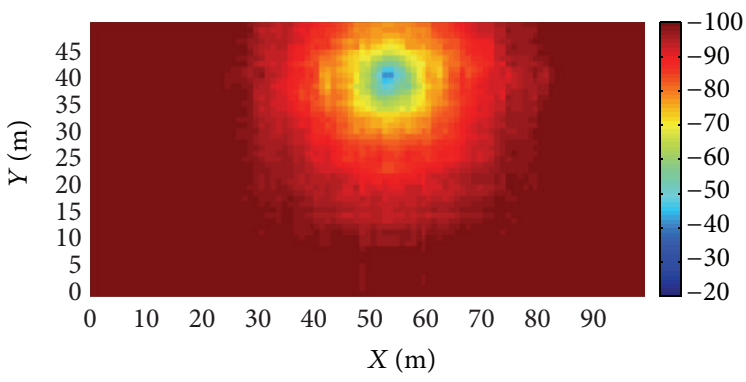

(f) SLR

Figure 2: The original radio map and the constructed results by different methods in the simulated experiments.

with $20 \%$ samples by different methods are shown in Figure 2. Here the parameters are $\lambda=0.08, \eta=1.0$, and $\sigma=$ 8. The experimental results show that the result of BLR is bad with block and gap, while the results of the proposed SLR method (Figure 2(f)) and IDW (Figure 2(c)) are more similar to the original measurements (Figure 2(a)). To get more precise results, the construction error is computed in a different sampling rate. The result is shown in Figure 3(a). From the results, it can be found that SLR and IDW yield good performance. As the noise has heavy effects on fingerprint, the radio map construction, and the positioning performance, the experiments are also implemented with different variances of noise. The result (Figure 3(b)) indicates that SLR and IDW behave robustly, while other methods provide bad results when the noise variance increases. It is denoted that the construction error is defined as the mean difference between the original and the constructed values at the reference points which are not included in the samples used for radio map construction.

In the above experiments, it is important to select suitable parameters $\lambda$ and $\eta$ for the minimum optimization model.
In practice, the optimal parameters can be figured out from the statistics of the errors between the original and the constructed results. Concretely, given a subset of the measurements (e.g., a random subset of $20 \%$ measurements), we can construct the whole measurement matrix by the SLR method. Thus, the construction error can be computed at all measurement points. For different parameters, the experiments are implemented and the average construction errors can be computed to get optimal parameters. The statistics of the construction errors with different $\lambda$ in $20 \%$ sampling are shown in Figure 5. The construction errors of all points and the points in $B$, which are used for SLR complement, are both computed. It is shown that if there is no smooth constraint, that is, $\lambda=0$, the construction errors of the points in $B$ are minor, but the errors of all points are large. In other words, the BLR method meets perfectly the $\mathscr{A}(X)=B$ condition, but the whole construction error is not acceptable, as the local consistence of RSSs is invalid. So the function of the smoothing parameter $\lambda$ is to balance the two factors, and the optimal $\lambda$ in our experiments is set to 0.008 . The optimal parameter $\eta$ can be obtained in a similar 


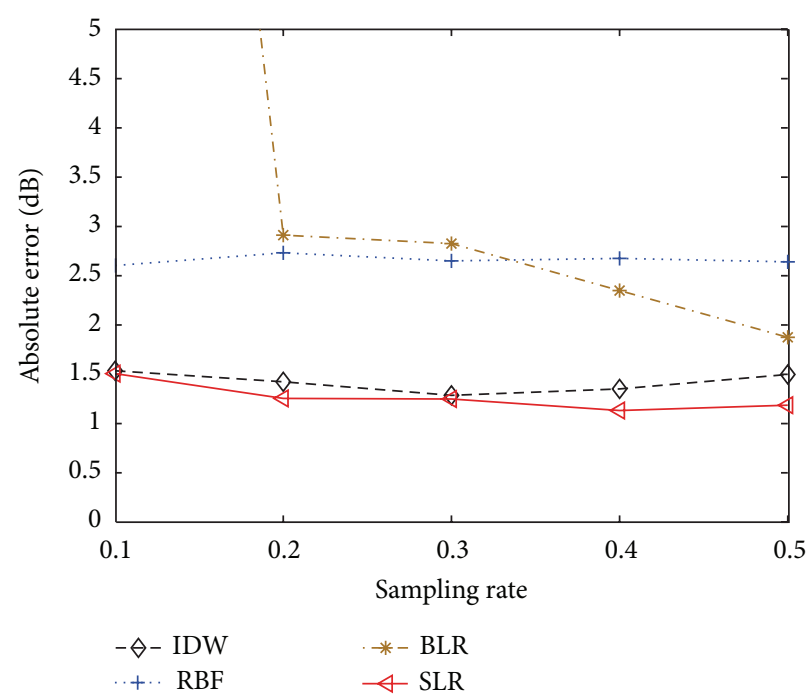

(a) Different sampling rates

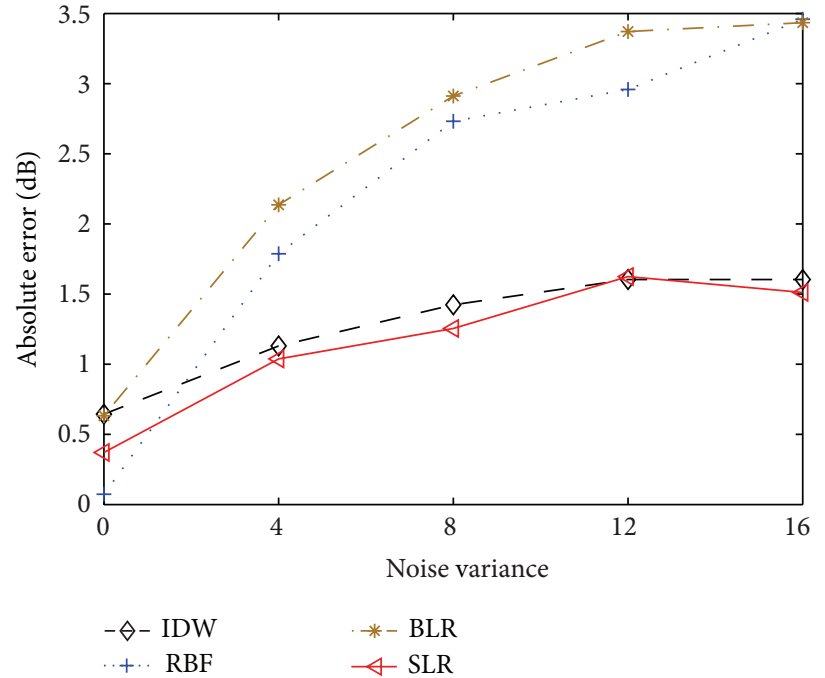

(b) Different variances of noise

FIGURE 3: Radio map construction errors on the simulated data.

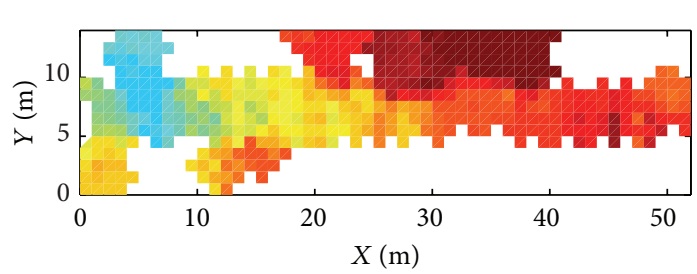

(a) IDW

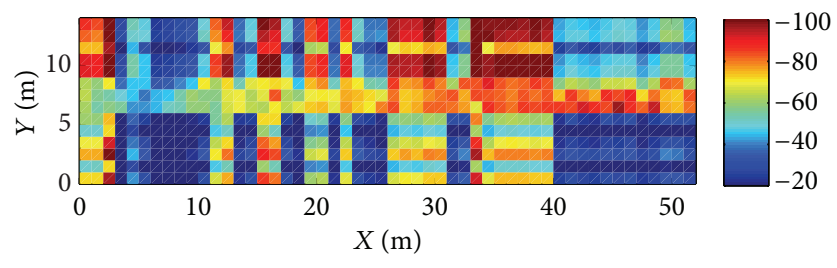

(c) BLR

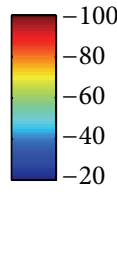

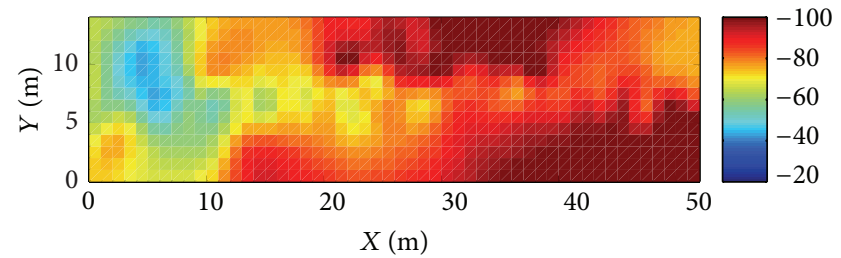

(b) RBF

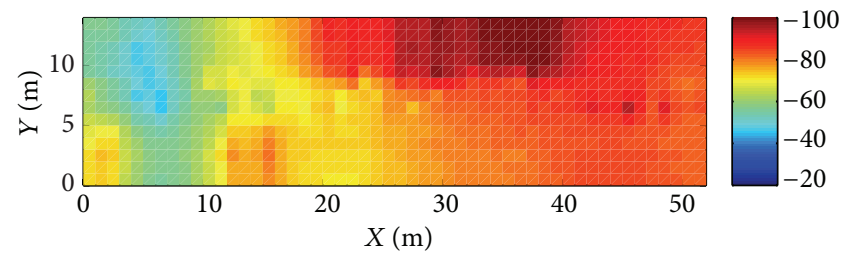

(d) SLR

FIGURE 4: Radio maps constructed results by different methods with 150 reference points on the real data.

procedure. It is denoted that if the experimental environment is changed, the parameters should be adjusted to get ideal constructed results.

In the real instance, the constructed fingerprint radio map of an AP using 50\% measurements of 337 reference points is shown in Figure 4. It is shown that the distribution of the RSSs in real environments is very complex compared with the simulation. From the constructed images, it can be found that the result of SLR method is continuous while the results of other methods are discontinuous. Although there are no complete $15 \times 53$ measurements, the constructed error can be computed by the average difference of the rest known measurements of the 337 reference points. The construction errors with different numbers of reference points are shown in Figure 6. Here, the reference points are randomly selected from the 337 points for radio map construction. It is shown that the construction error of the BLR method is much larger than others, so it is not included in Figure 6. From the results, it is concluded that the proposed SLR method provides the best performance, though the errors are bigger than those in simulated situation. To get more practical results, the construction experiments are repeated 5 times for a fixed number of reference points and the mean error of the 5 experiments is regarded as the final result for the number. For convenience, the parameter $\lambda$ is also set to 0.08 and the parameter $\eta$ is set to 1.0 in these experiments. As the actual noise of the real measurement is unknown, the noise variation experiments are not implemented on the real data.

4.3. Positioning Results. To further evaluate the efficiency of the constructed radio maps, these constructed results are used for positioning. Here we adopt the widely used positioning method, the kernel-based algorithm [3], in the 


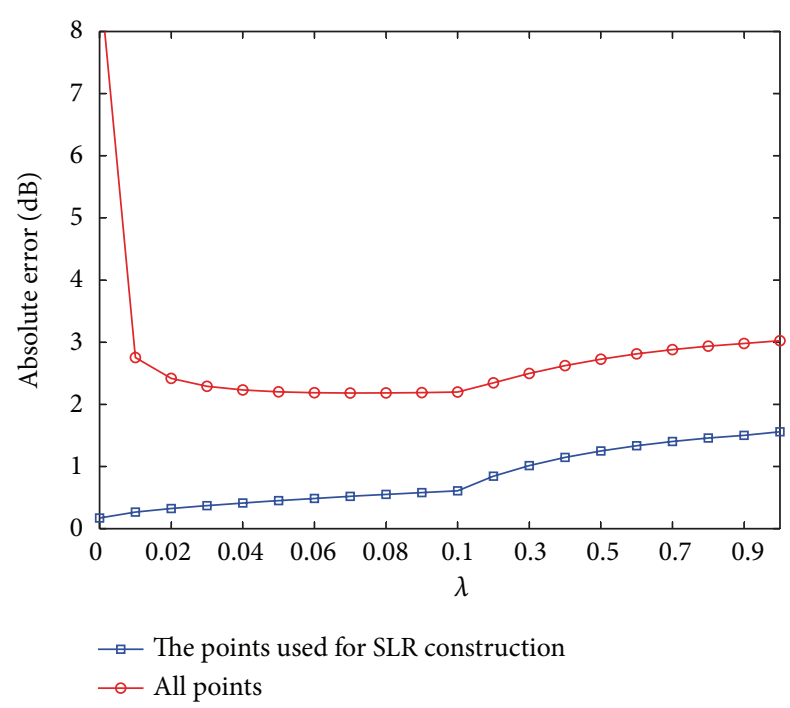

Figure 5: The construction errors of different smoothing parameters.

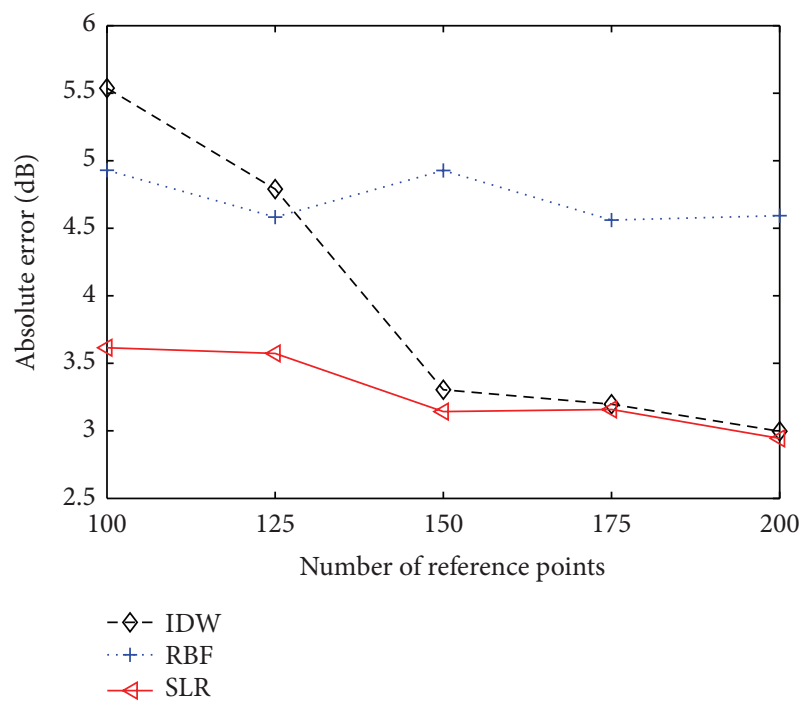

FIgURE 6: Radio map construction errors on the real data.

online positioning phase. In our experiments, the estimated location is the mean location of 20 nearest neighbors in the sense of RSS value similarity. In the simulated situation, parts of measurements of the reference points are used for the radio map construction while the rest are served as the test. The positioning error is defined as the average distance between the estimated location and its real location. Figure 7(a) shows the positioning errors of the radio maps at different sampling rates. The results indicate that the SLR methods yield the best performance, especially at low sampling rate. For example, the SLR constructed method gets $1.07 \mathrm{~m}$ precision at $20 \%$ sampling rate while the other methods cannot get the same accuracy even at $50 \%$ sampling rate. The constructed radio maps with different noises are also used for positioning and the results are shown in Figure 7(b). It shows that

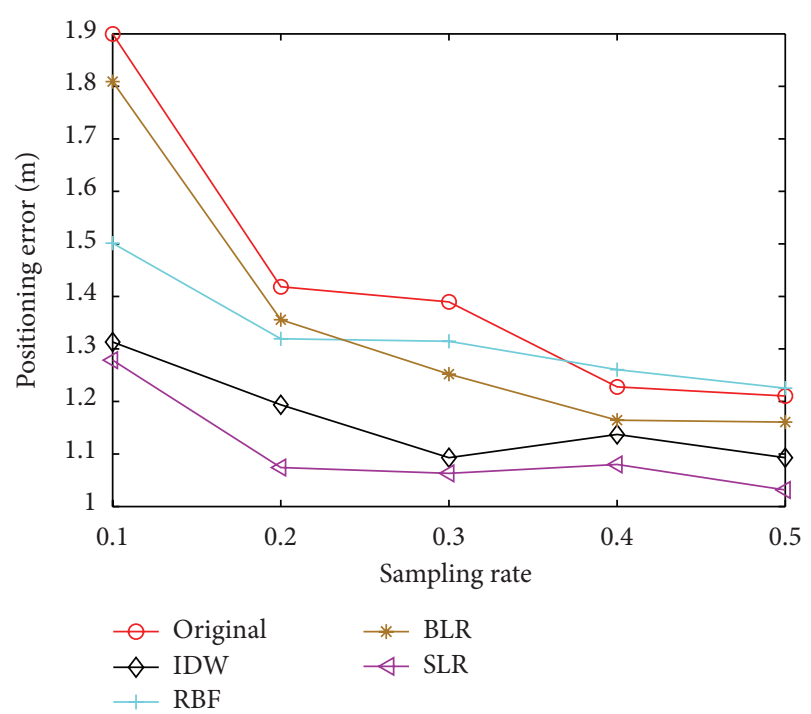

(a) Different sampling rates

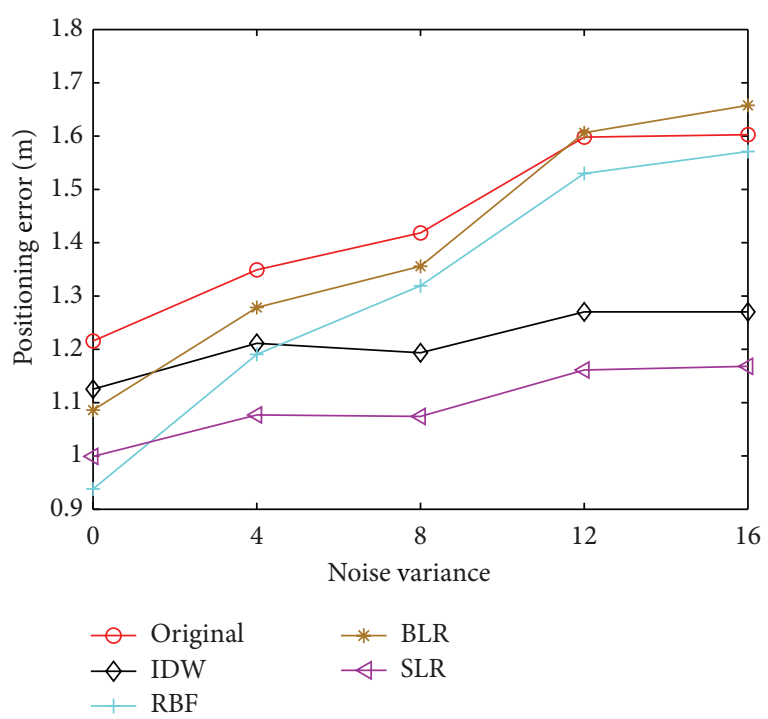

(b) Different variances of noise

FIgURE 7: Positioning errors on the simulated data.

the positioning results are consistent with the radio map construction results. The positioning performance of the SLR method has robust behavior.

In the real environment, the constructed fingerprint radio maps with different numbers of reference points are also used for positioning. The positioning errors are shown in Figure 8. Compared with the other methods, the result of the SLR method has the best positioning accuracy, and for the same positioning accuracy, the SLR method needs less RSS measurements. For example, the SLR method provides $1.53 \mathrm{~m}$ precision using 150 reference points while others require 200 reference points at least. So the SLR method yields a more efficient radio map construction solution. It is denoted that the positioning errors are also the average values of 5 times positioning results. 


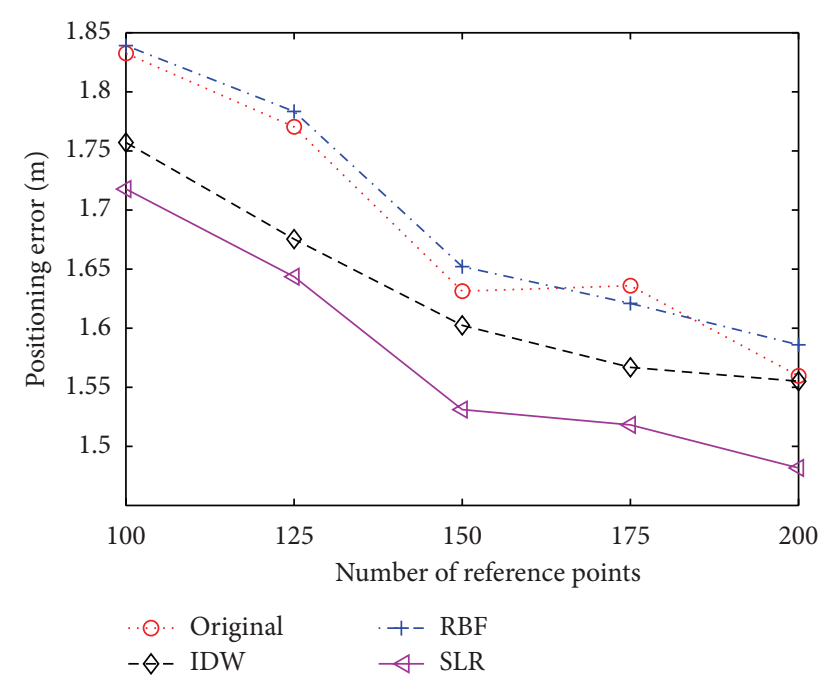

FIgURE 8: Positioning errors on the real data.

\section{Conclusion}

In this paper, an efficient radio map construction method is proposed based on low-rank approximation. Observing that the signal has high spatial correlation, the RSS distribution is modeled as low-rank matrix. By the low-rank completion theory, given relative sparse entries, the whole measurement matrix is recovered using an optimization method. As the basic low-rank completion model does not preserve the consistence of the radio map, a revised low-rank model is proposed to get practical results, in which the consistence is represented as the smooth term in the model. The solution of the proposed model is also discussed in detail. The proposed method is validated through both simulation and real experiments. The results of the experiments show that the proposed method significantly reduces the number of measurements for radio map construction and behaves robustly to noise interference. The constructed radio maps are also used for positioning. It is shown that the positioning accuracy is improved compared with the state-of-the-art methods. In future works, we plan to explore the radio map updating mechanisms and apply the proposed method in complicated environment.

\section{Acknowledgments}

This paper is partly supported by the 973 Program of China (no. 2011CB302703), the National Natural Science Foundation of China (nos. 61133003, 61171169, and 61170103), and the Beijing Natural Science Foundation (no. kz201310005006).

\section{References}

[1] P. Bahl and V. N. Padmanabhan, "RADAR: an in-building RFbased user location and tracking system," in Proceedings of the 19th Annual Joint Conference of the IEEE Computer and Communications Societies (INFOCOM '00), pp. 775-784, Tel Aviv, Israel, March 2000.
[2] M. A. Youssef, A. Agrawala, and A. U. Shankar, "WLAN location determination via clustering and probability distributions," in Proceedings of the 1st IEEE International Conference on Pervasive Computing and Communications (PerCom '03), pp. 143-150, Fort Worth, Tex, USA, March 2003.

[3] A. Kushki, K. N. Plataniotis, and A. N. Venetsanopoulos, "Kernel-based positioning in wireless local area networks," IEEE Transactions on Mobile Computing, vol. 6, no. 6, pp. 689705, 2007.

[4] D. Madigan, E. Elnahrawy, R. P. Martin, W.-H. Ju, P. Krishnan, and A.S. Krishnakumar, "Bayesian indoor positioning systems," in Proceedings of the 24th Annual Joint Conference of the IEEE Computer and Communications Societies (INFOCOM '05), pp. 1217-1227, Miami, Fla, USA, March 2005.

[5] H. Ji, C. Liu, Z. Shen, and Y. Xu, "Robust video denoising using Low rank matrix completion," in Proceedings of the IEEE Computer Society Conference on Computer Vision and Pattern Recognition (CVPR '10), pp. 1791-1798, San Francisco, Calif, USA, June 2010.

[6] J. Cheng, H. Jiang, X. Ma et al., "Efficient data collection with sampling in WSNs: making use of matrix completion techniques," in Proceedings of the 53rd IEEE Global Communications Conference (GLOBECOM '10), pp. 1-5, Miami, Fla, USA, December 2010.

[7] E. Candes and B. Recht, "Exact matrix completion via convex optimization," Communications of the ACM, vol. 55, no. 6, pp. 111-119, 2012.

[8] Z. Xiang, H. Zhang, J. Huang, S. Song, and K. C. Almeroth, "A hiddenenvironment model for constructing indoor radio maps," in Proceedings of the 6th IEEE International Symposium on a World of Wireless Mobile and Multimedia Networks, pp. 395-400, Taormina-Giardini Naxos, Italy, June 2005.

[9] S. P. Kuo and Y. C. Tseng, "Discriminant minimization search for large-scale RF-based localization systems," IEEE Transactions on Mobile Computing, vol. 10, no. 2, pp. 291-304, 2011.

[10] J. Krumm and J. Platt, "Minimizing calibration effort for an indoor 802.11 device location measurement system," Tech. Rep. MSRTR-2003-82, Microsoft Research, 2003.

[11] C. Feng, W. Au, S. Valaee, and Z. Tan, "Received signal strength based indoor positioning using compressive sensing," IEEE Transaction on Mobile Computing, vol. 11, no. 12, pp. 1983-1993, 2011.

[12] E. J. Candès, X. Li, Y. Ma, and J. Wright, "Robust principal component analysis?" Journal of the ACM, vol. 58, no. 3, article 11, 2011.

[13] E. J. Candès and Y. Plan, "Tight oracle inequalities for lowrank matrix recovery from a minimal number of noisy random measurements," IEEE Transactions on Information Theory, vol. 57, no. 4, pp. 2342-2359, 2011.

[14] D. Zachariah, M. Sundin, M. Jansson, and S. Chatterjee, "Alternating least-squares for low-rank matrix reconstruction," IEEE Signal Processing Letters, vol. 19, no. 4, pp. 231-234, 2012.

[15] Y. Zhang, M. Roughan, W. Willinger, and L. Qiu, "Spatiotemporal compressive sensing and internet traffic matrices," in Proceedings of the ACM Conference on Data Communication (SIGCOMM '09), pp. 267-278, Barcelona, Spain, August 2009.

[16] S. Nikitaki, G. Tsagkatakis, and P. Tsakalides, "Efficient training for fingerprint based positioning using matrix completion," in Proceedings of 20th European Signal Processing Conference (EUSIPCO '12), pp. 195-199, Bucharest, Roumania, August 2012. 
[17] B. Recht, M. Fazel, and P. A. Parrilo, "Guaranteed minimumrank solutions of linear matrix equations via nuclear norm minimization," SIAM Review, vol. 52, no. 3, pp. 471-501, 2010. 


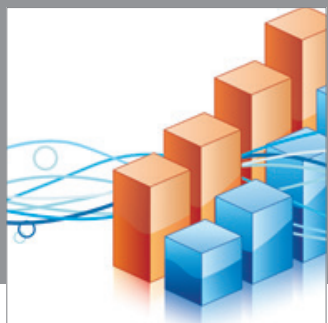

Advances in

Operations Research

mansans

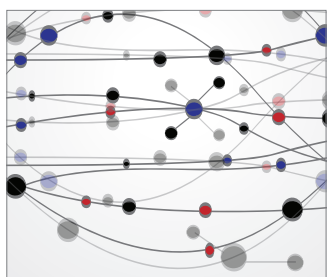

The Scientific World Journal
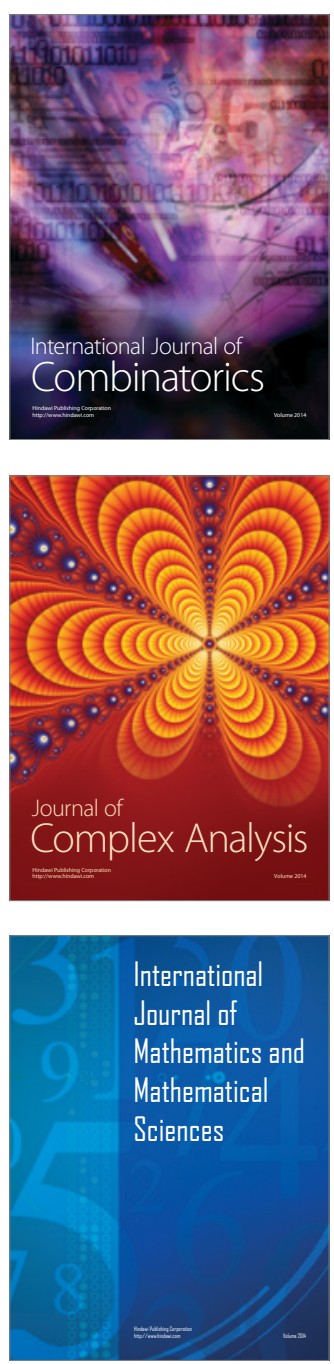
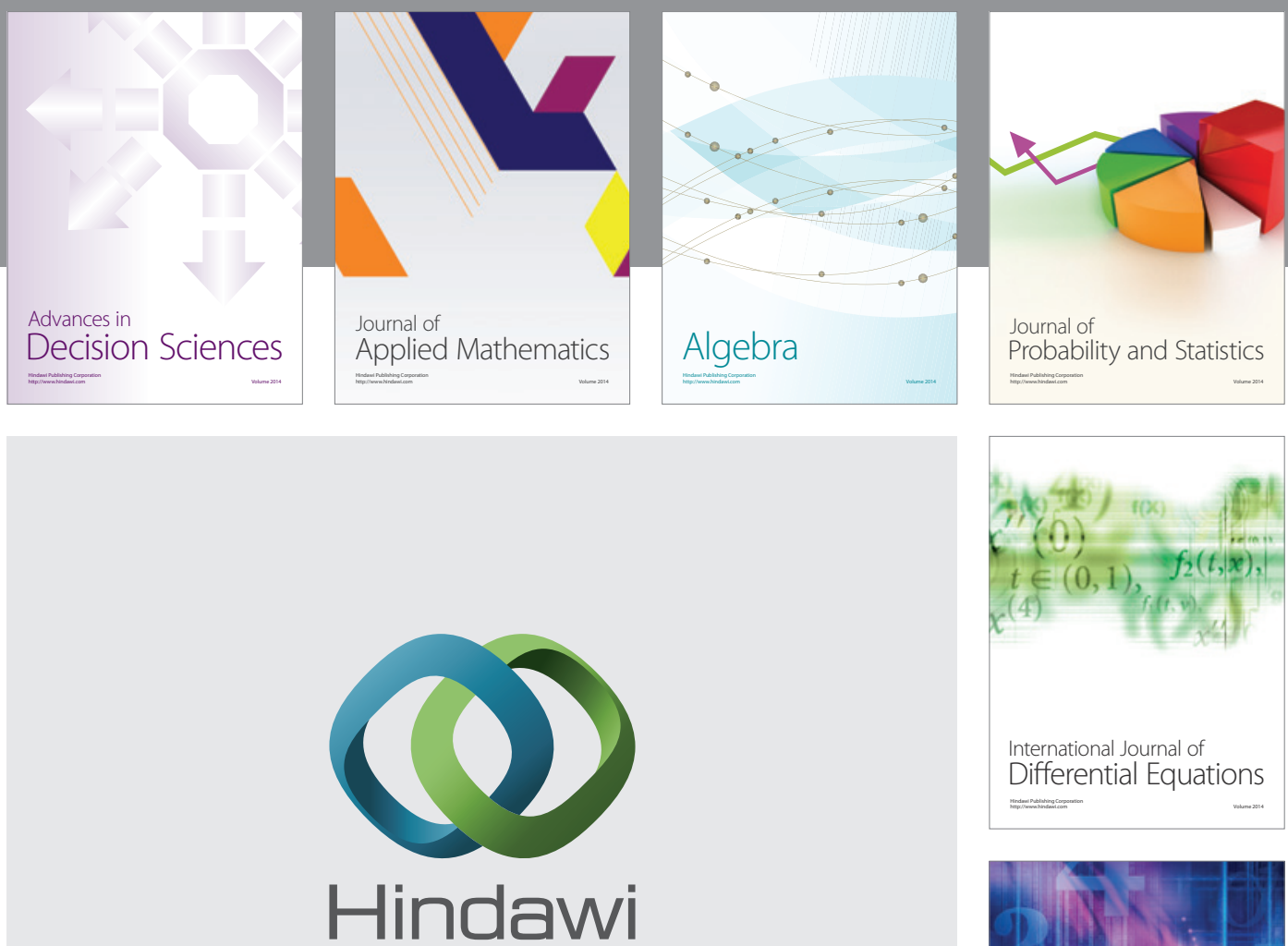

Submit your manuscripts at http://www.hindawi.com
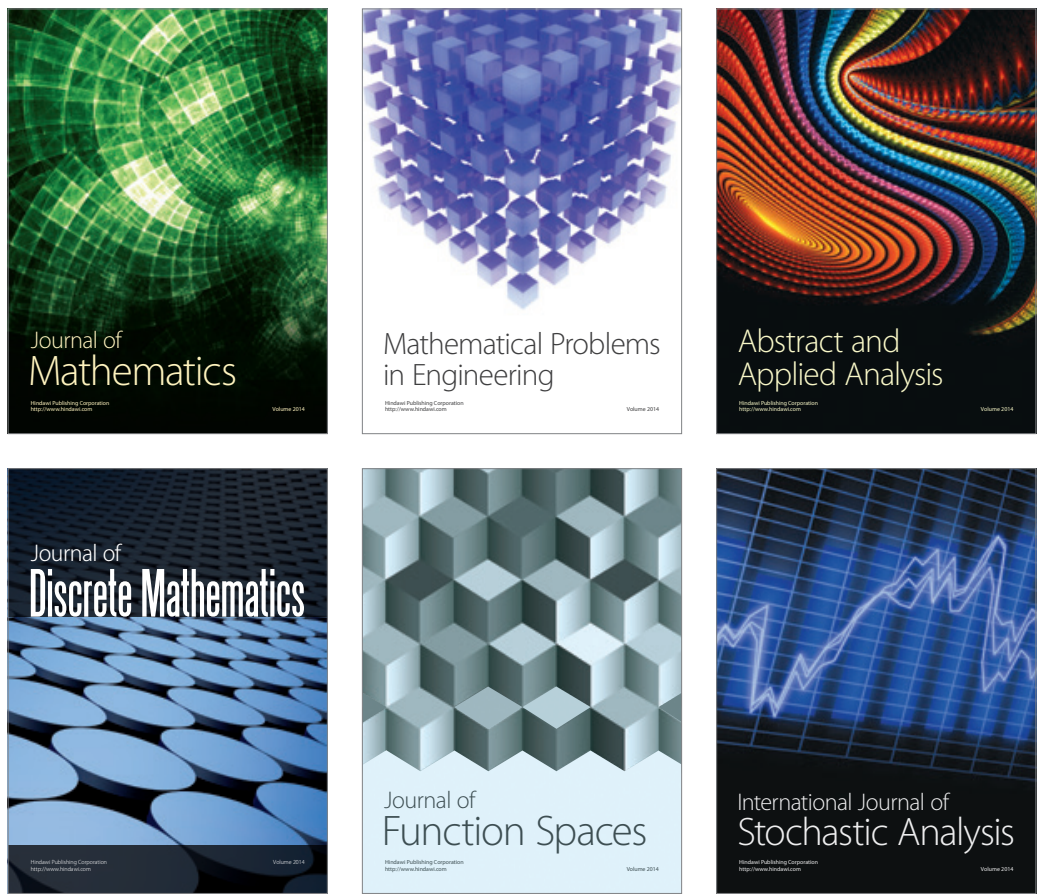

Journal of

Function Spaces

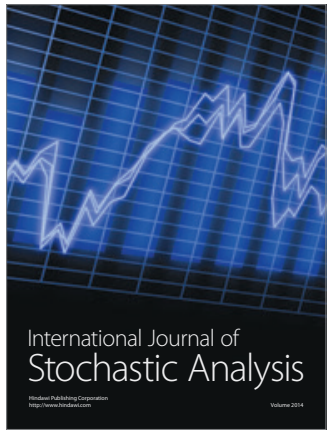

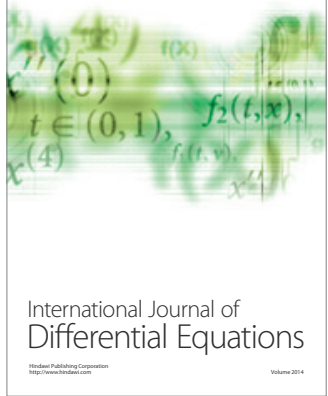
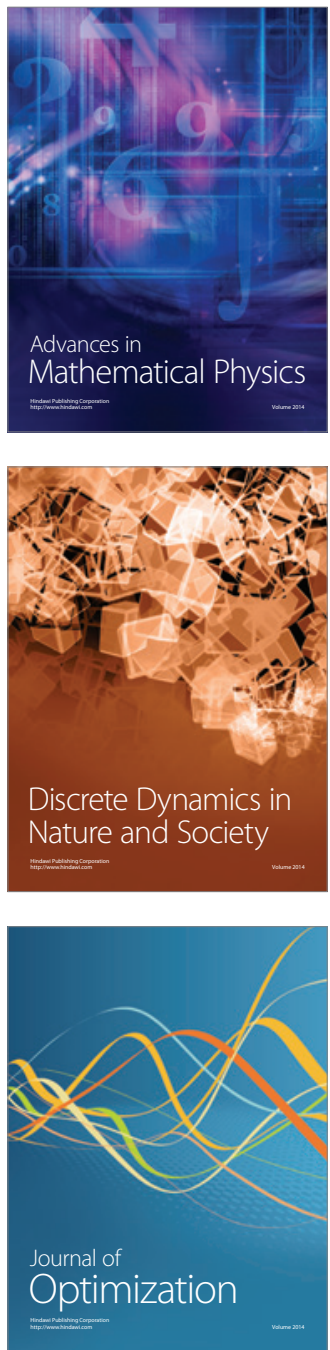